\title{
(6) OPEN ACCESS \\ Preventing Australian football injuries with a targeted neuromuscular control exercise programme: comparative injury rates from a training intervention delivered in a clustered randomised controlled trial
}

\author{
Caroline F Finch, ${ }^{1}$ Dara M Twomey, ${ }^{2}$ Lauren V Fortington, ${ }^{1}$ Tim L A Doyle, ${ }^{3}$ \\ Bruce C Elliott, ${ }^{4}$ Muhammad Akram, ${ }^{1}$ David G Lloyd ${ }^{3,4}$
}

\begin{abstract}
- Additional material is published online only. To view please visit the journal online (http://dx.doi.org/10.1136/ injuryprev-2015-041667).

${ }^{1}$ Australian Centre for Research into Injury in Sport and its Prevention (ACRISP), Federation University Australia, Ballarat, Victoria, Australia

${ }^{2}$ Faculty of Health, Federation University Australia, Ballarat, Victoria, Australia

${ }^{3}$ Centre for Musculoskeletal Research, Menzies Health Institute Queensland, Griffith University, Gold Coast, Queensland, Australia ${ }^{4}$ School of Sport Science, Exercise and Health, the University of Western Australia, Perth, Western Australia, Australia
\end{abstract}

\section{Correspondence to} Professor Caroline F Finch, Australian Centre for Research into Injury in Sport and its Prevention (ACRISP), Federation University Australia, SMB Campus, PO Box 663, Ballarat VIC 3353 Australia; c.finch@federation.edu.au

Received 27 April 2015 Revised 11 August 2015 Accepted 24 August 2015 Published Online First 23 September 2015
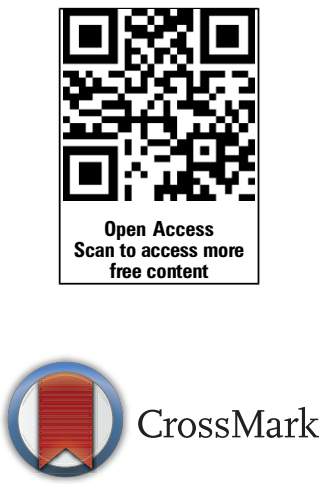

To cite: Finch $C F$, Twomey DM, Fortington LV, et al. Inj Prev 2016;22. 123-128.

\section{ABSTRACT}

Background Exercise-based training programmes are commonly used to prevent sports injuries but programme effectiveness within community men's team sport is largely unknown.

Objective To present the intention-to-treat analysis of injury outcomes from a clustered randomised controlled trial in community Australian football.

Methods Players from 18 male, non-elite, community Australian football clubs across two states were randomly allocated to either a neuromuscular control (NMC) (intervention $n=679$ players) or standard-practice (control $n=885$ players) exercise training programme delivered as part of regular team training sessions $(2 \times$ weekly for 8-week preseason and 18-week regularseason). All game-related injuries and hours of game participation were recorded. Generalised estimating equations, adjusted for clustering (club unit), were used to compute injury incidence rates (IIRs) for all injuries, lower limb injuries (LLIs) and knee injuries sustained during games. The IIRs were compared across groups with cluster-adjusted Injury Rate Ratios (IRRs).

Results Overall, 773 game injuries were recorded. The lower limb was the most frequent body region injured, accounting for $50 \%$ of injuries overall, $96(12 \%)$ of which were knee injuries. The NMC players had a reduced $\mathrm{LLI}$ rate compared with control players (IRR: $0.78(95 \% \mathrm{Cl} 0.56$ to 1.08$), p=0.14$.) The knee IIR was also reduced for NMC compared with control players (IRR: $0.50(95 \% \mathrm{Cl} 0.24$ to 1.05), $p=0.07)$.

Conclusions These intention-to-treat results indicate that positive outcomes can be achieved from targeted training programmes for reducing knee and LLI injury rates in men's community sport. While not statistically significant, reducing the knee injury rate by $50 \%$ and the $\mathrm{LLI}$ rate by $22 \%$ is still a clinically important outcome. Further injury reductions could be achieved with improved training attendance and participation in the programme.

\section{INTRODUCTION}

Team-based exercise training programmes have become a popular method for delivering injury prevention at the club level in many sports. Until recently, evaluation of the benefits of such programmes has largely been undertaken in efficacy studies involving highly controlled or laboratorybased settings, thereby providing limited knowledge of real-world benefits. ${ }^{1}$ Since the gap in research demonstrating the effectiveness of these programmes was recognised, there has been an increase in the number of investigations directly in the non-elite, community sport setting. ${ }^{2}$ Recent systematic reviews summarising the effectiveness of training programmes for reducing injuries in team sport have identified varying outcomes for injury reduction. $^{3-6}$ The variation is attributed to a range of factors, including participant age and sex, level of competition, the type of components included in the programme and compliance with the programme. As such, specific investigations towards understanding the effectiveness of injury prevention training programmes for different sports settings is now required.

Australian football (AF) is one of the most popular team sports in Australia. At the community sport level, AF is played by all age groups, men and women. Adult male participants (generally between 18 years and 30 years old) commonly play in a weekend game, with training sessions once or twice weekly over 5-6 months of the year. The game is played at a fast pace, with players subject to continuous running, bursts of sprinting, sudden changes of direction, frequent jumping/landing and heavy physical contact. These characteristic movements of AF also present players with a high risk of injury. ${ }^{7} 8$ Although the collection and quality of injury data in community $\mathrm{AF}$ is limited, ${ }^{9}$ the burden of AF injury is large, it being ranked one of the highest of all team sports leading to hospital treated injury. ${ }^{10}$ The lower limb is the most common body region injured in AF with the knee consistently reported as one of the most frequently injured body parts. ${ }^{9-11}$ These injuries have high personal costs, leading to reduced or ceased participation, long-term degenerative joint damage and ongoing pain. ${ }^{12-14}$ Interventions aimed at preventing all lower limb injuries (LLIs) are needed to support the long-term health and well-being of players and enable promotion of the positive health benefits gained from participation.

The Preventing Australian Football Injuries through eXercise (PAFIX) study was a clustered randomised controlled trial (cRCT) in community AF aimed at reducing the number of LLIs, particularly knee injuries. ${ }^{15}$ Players from clubs assigned to the intervention arm undertook a programme of progressive, targeted exercises as part of their regular training sessions (a neuromuscular control (NMC) programme), beginning in the preseason and continuing throughout the competition-season. 
The second group (control) of clubs/players was provided with a sham exercise programme that replicated common training practices in community AF.

This paper presents the intention-to-treat (ITT) analysis of the cRCT results in relation to whether the training programme led to a reduction in the rate of knee injuries or LLIs in games.

\section{METHODS}

This study was designed according to the CONSORT statement (refer to online supplementary appendix 1$).{ }^{16}$ The study rationale, design and full protocol have been previously published. ${ }^{15}$ The specific content and delivery of the trialled exercise training programme content have also been published. ${ }^{17}$ Minor amendments to the original study protocol ${ }^{15}$ were needed to adjust for procedural challenges that were encountered when implementing the trial. These amendments, structured around the CONSORT statement components, ${ }^{16}$ are outlined in online supplementary appendix 2 and all published and procedural documents are available online (http://www.pafixproject.wordpress.com).

\section{Inclusion and exclusion criteria}

Community male AF clubs from two Australian states participated in the PAFIX trial over an 8 -week preseason period and an 18-week playing season (26 weeks total), in 2007 or 2008 . Eighteen clubs (nine in 2007 and nine in 2008) were included, resulting in 2017 players assessed for inclusion eligibility. Figure 1 shows the allocation of players to trial arms and the numbers of players involved in the final trial and its analysis.
Full details of the inclusion and exclusion criteria are presented in online supplementary appendix 2. In short, players were included if they were registered to play for one of the participating teams, were at least 18 years of age and attended at least one training session in the first 13 weeks of the programme. Players from either group who did not train in the first 13 weeks (the point of transition from early season to midseason) were excluded from analyses as they had not been exposed to a sufficient degree of the intervention; this was determined to be a reasonable failure-to-start exclusion that still met the criteria of an ITT analysis. ${ }^{18}$

The total number of players recruited into PAFIX was 1691. Of these, 751 were allocated to the NMC group with 679 retained for analysis (ie, met all inclusion criteria) and 940 players were allocated to the control group, from which 885 were retained. The total number of players included for analysis was 1564 (figure 1).

\section{Intervention}

Players from the NMC and control groups participated in a set of training exercises as part of their regular training programme twice per week. ${ }^{17}$ The researchers trained team-based primary data collectors (PDCs) to deliver the prescribed exercises to the participating players. The NMC group received a programme of evidence-based neuromuscular and biomechanical exercises specifically targeted at reducing LLI, while the control group was provided with a 'sham' programme of exercises similar to those regularly undertaken at training. ${ }^{17}$ Neither the players, clubs
Figure 1 Allocation of players to interventions in the PAFIX trial. Non-attendance at training sessions meant no exposure at all to any of the trial conditions so players were removed. NMC, neuromuscular control; PAFIX, Preventing Australian Football Injuries through eXercise.

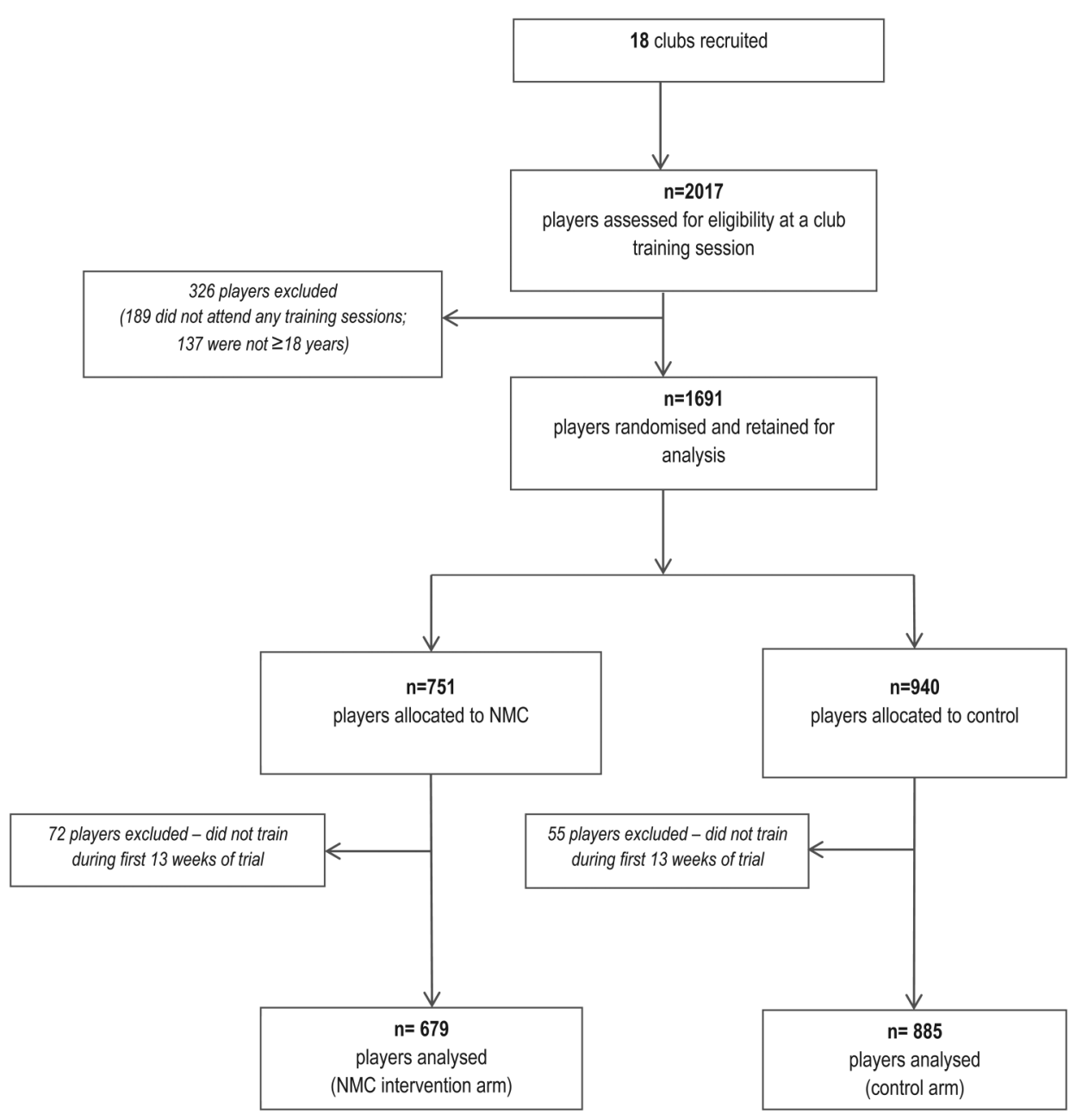


nor PDCs were aware of what programme those in the other study condition received.

\section{Data collection and coding}

Each player participated over a 26-week period in 2007 or 2008. In this time, the PDC recorded the number of training sessions attended, the number of games played, the number of game hours played and the number of injuries sustained. An injury was defined as something that caused a player to seek medical attention (on or off the field) or to leave the field of play. Injury details were recorded by the PDC from observation and later confirmed with the player and medical staff where available. The injury definition and data collection procedures have been described elsewhere and shown to be reliable. ${ }^{19}$ Training sessions in $\mathrm{AF}$ last for around $1.5 \mathrm{~h}$ with both programmes designed to take no more than the first $20 \mathrm{~min}$ of that time.

Consistent with the CONSORT statement, ${ }^{16}$ an ITT analysis was performed. Injury incidence rates (IIRs) for all injury, all LLI and knee injury only were calculated for all players combined, as well as separately for the NMC and control groups. The estimated IIR was calculated as the number of injuries sustained divided by the exposure measure (game hours played.) For each IIR, a 95\% CI was calculated assuming Poisson errors. The IIRs in the NMC (IIR-NMC) and control (IIR-CONTROL) groups were compared by calculating the IRR as $\mathrm{IRR}=\mathrm{IIR}-\mathrm{NMC} / \mathrm{IIR}-\mathrm{CONTROL}$ and the $95 \% \mathrm{CI}$ as , where SE $(\ln (\mathrm{IRR}))$ is the IRR's SD.

To adjust for clustering effects (measured by the intraclass correlation (ICC)) associated with the sampling of teams within the clubs or states that may have influenced injury rates, a Generalised Estimating Equation (GEE) with Poisson distribution, log link function and an exchangeable autocorrelation structure was fitted to the injury rate data. The GEE assessed the effectiveness of the training programme in reducing the rate of injuries in games. Specifically, assessment of reduction in knee injuries sustained during games was undertaken as that was how the study was initially powered. Since the data were somewhat zero-inflated, a GEE with negative binomial distribution and exchangeable correlation structure was also fitted to account for overdispersion. As this obtained similar results to the first GEE model its results are not presented. The dependent variable was the player-specific IIR, with the trial arm included as an indicator factor and the football club effect nested within the state factor to account for clustering effects. Results for the GEE models are expressed as IRRs with 95\% CIs and interpreted as the percentage change in IIR between the NMC and the control groups. To further confirm the modelling results, bootstrapping (which used repeated draws with replacement from the observed sample to create simulated samples) was undertaken and no significant difference to the main GEE results was found. Post hoc power calculations were based on Hayes' method. ${ }^{20}$

All data were originally double-entered into a database. Analyses were performed in statistical packages Stata (V.12.1) and R (R Core Team 2013, http://www.r-project.org, V.3.1.2). The statistical analyses were performed by author MA blinded to the intervention arm allocation.

\section{RESULTS}

\section{Trial group characteristics}

Each trial arm had nine clubs, with 38 NMC teams and 59 control teams, spread across the two geographical regions and years (table 1). It was not possible to fully assess baseline equivalence of the two groups in terms of player age or level of play because that information was only obtained from a (nonrandom) subset of players.

A total of 1032 injuries were recorded in 563 players, of which 773 injuries were sustained during games. The lower limb was the most frequent body region injured, accounting for $50 \%$ of injuries overall. The upper leg was the most frequently injured body part (table 2).

\section{Injury incidence rate comparisons}

As expected, there were no significant differences in the all-injury IIR between the NMC and control groups. The game IIR in the NMC group was smaller than that for the control group and the associated cluster-adjusted IRR of 0.92 for game injuries indicates an $8 \%$ non-significant reduction in all injures for the NMC group (table 3).

There was a significantly smaller IIR for LLI in the NMC group compared with the control group in games but this was not significant after cluster adjustment. Nonetheless, the clusteradjusted IRR indicates a $22 \%$ reduction in the exposure-adjusted LLI IIR in the NMC group, compared with control players. The cluster-adjusted knee injury IRR was marginally not significant and indicated that knee injury rates were $50 \%$ lower in the NMC group, compared with control players.

For each injury category, the cluster adjustment led to smaller IIRs, in favour of the NMC intervention. However, CIs around them were larger, justifying the need for cluster adjustment. The observed overall rate of injuries, and the proportion of injuries that were to the knee, were both lower than what was used to justify the sample size calculations in the initial study protocol. For this reason, table 3 also includes post hoc power calculation for each of the cluster-adjusted IRRs.

\section{DISCUSSION}

This is the first study to report the effect of a targeted exercise programme on reducing injuries in game-related adult, male, community AF players. A lower, but not statistically significant, rate of knee injury and LLI overall was observed in players allocated to a specific programme of NMC training exercises compared with those allocated to a standard-practice sham exercise programme. Players who undertook the NMC programme had a $22 \%$ reduction in LLI rates and a $50 \%$ reduction in knee injury rates, when adjusted for exposure and clustering. These are important and highly clinically relevant outcomes given the frequency and associated costs of LLI arising from AF games, and the priority needs for prevention. ${ }^{9}$ With growing evidence

Table 1 Number of clubs, teams and players allocated to study arms in the PAFIX clustered randomised controlled trial

\begin{tabular}{|c|c|c|}
\hline & $\begin{array}{l}\text { Intervention arm } \\
\text { (neuromuscular } \\
\text { control programme) }\end{array}$ & $\begin{array}{l}\text { Control arm } \\
\text { (sham programme) }\end{array}$ \\
\hline Number of clubs & 9 & 9 \\
\hline Number of teams & 38 & 59 \\
\hline \multicolumn{3}{|c|}{ Number of teams by grade* } \\
\hline Seniors & 27 & 33 \\
\hline Reserves/Colts & 11 & 26 \\
\hline Number of players & 679 & 885 \\
\hline \multicolumn{3}{|c|}{$\begin{array}{l}\text { *Grade refers to the level of competitive play. 'Seniors' being a better standard of } \\
\text { playing competition and 'Reserves/Colts' being a lower level standard. In Reserves/ } \\
\text { Colts, players tend to be slightly younger than players in Seniors teams. (Colts is an } \\
\text { under } 19 \text { years competition in one region-but only those } 18+\text { years are included in } \\
\text { this trial, as per the protocol inclusion criteria). } \\
\text { PAFIX, Preventing Australian Football Injuries through Exercise. }\end{array}$} \\
\hline
\end{tabular}


Table 2 Proportion of game injuries by body region in players in the PAFIX clustered randomised controlled trial by study arm

\begin{tabular}{|c|c|c|c|}
\hline \multirow[b]{2}{*}{$\begin{array}{l}\text { Proportion of injuries by body } \\
\text { region* }\end{array}$} & \multicolumn{3}{|l|}{ Study arm } \\
\hline & $\begin{array}{l}\text { All players } \\
\mathrm{n}=773 \\
\text { injuries }\end{array}$ & $\begin{array}{l}\text { NMC arm } \\
\mathrm{n}=335 \\
\text { injuries }\end{array}$ & $\begin{array}{l}\text { Control arm } \\
\mathrm{n}=438 \\
\text { injuries }\end{array}$ \\
\hline Lower limb totalt & 50.2 & 46.3 & 53.2 \\
\hline Upper leg & 17.2 & 15.8 & 23.9 \\
\hline Knee & 12.0 & 9.6 & 13.9 \\
\hline Lower leg & 8.2 & 4.8 & 10.7 \\
\hline Ankle/foot/toes & 12.8 & 16.1 & 10.3 \\
\hline Head/neck total† & 17.9 & 23.3 & 13.7 \\
\hline Head and neck & 7.1 & 8.7 & 5.9 \\
\hline Face/teeth/mouth & 10.7 & 14.6 & 7.8 \\
\hline Upper limb total† & 17.5 & 14.0 & 20.1 \\
\hline $\begin{array}{l}\text { Shoulder/upper arm/elbow/lower } \\
\text { arm }\end{array}$ & 9.7 & 9.6 & 9.8 \\
\hline Hand/finger & 7.8 & 4.5 & 10.3 \\
\hline Trunk totalt & 14.2 & 15.8 & 13.0 \\
\hline Trunk/abdomen/chest/back/glutes & 8.9 & 9.9 & 8.2 \\
\hline Groin/Hip & 5.3 & 6.0 & 4.8 \\
\hline Other & 0.3 & 0.6 & 0.0 \\
\hline
\end{tabular}

of the effectiveness of targeted exercise training programmes for injury prevention in other sports, this study provides the first support for such programmes in men's community AF.

The NMC programme was associated with improved LLI and knee injury rates. There was no significant difference in the all-injury rate across the two trial arms. This was to be expected as the exercise components and the PAFIX trial were specifically designed to address knee injury rather than all types of injury. ${ }^{15}$ Since the PAFIX trial was first designed, it has become apparent that the same intervention exercise programme components could potentially reduce the risk of all LLIs, not just knee injuries. ${ }^{3}$ For this reason, this paper considers the rate reductions in both groups of injury types.
The injury patterns reported in this study are generally consistent with those reported in previous studies of men's AF with the most frequent injured body parts being the upper leg/thigh and knee. ${ }^{9}{ }^{10}$ However, the proportion of all injuries to the knee was lower than that expected when the study sample size calculations were made $(12 \%$ of all observed injuries, compared with the anticipated 33\%). Post hoc power calculations indicate that the study was underpowered for all comparisons, but least so for the comparison of knee injury rates. Nonetheless, the observed reductions in knee injuries are highly significant from a clinical sports medicine point of view and have important implications for the design of future exercise training programmes in AF.

A number of features of injury prevention programmes influence injury outcomes and these can vary according to the participants of the study (eg, sex, age, level of play). ${ }^{21} 22$ Most previous trials of the benefits of injury prevention exercise programmes in sport have been undertaken in female youth participating in soccer, ${ }^{23} 24$ with just a few focused on adult male participants at a community (non-elite) level. ${ }^{6}{ }^{22}$ It is important to establish effectiveness in specific participant populations such as men as they comprise the majority of participants in many sports and certainly in AF. The current study adds new evidence about the effectiveness of injury prevention programmes in male community sports participants.

It is important for players to be compliant with prevention programmes for them to reduce injury rates. ${ }^{25} 26$ Given that many players in PAFIX did not train regularly and most did not attend the two weekly sessions intended as part of the programme, ${ }^{27}$ the ITT assessment of reduction in LLI rates in games is likely to have underestimated the true effect of the NMC programme. It is possible that a stronger intervention effect might have been identified if we had employed a perprotocol analysis $^{28}$ and this will be of interest in future work. While the frequency of exposure to the training programme could be quantified, ${ }^{27}$ the quality of the actual exercise performance and programme fidelity were not formally measured. Ideally, frequency and quality of training should be captured, and linked to the injury outcomes, to provide stronger evidence of a connection between players who train, those who train well and players who do or do not get injured. ${ }^{29}$

Injury prevention programmes should have specific components or exercises that effectively target LLI, including knee

Table 3 Comparison of game related injury rates in the NMC intervention and sham (control) PAFIX trial groups

\begin{tabular}{|c|c|c|c|c|c|c|c|}
\hline & $\begin{array}{l}\text { Number } \\
\text { of injuries }\end{array}$ & $\begin{array}{l}\text { Number of } \\
\text { hours spent } \\
\text { in games }\end{array}$ & $\begin{array}{l}\text { IIR per } 1000 \text { game hours } \\
\text { (95\% confidence limits)* }\end{array}$ & $\begin{array}{l}\text { IRR } \\
\text { (95\% confidence limits) } \\
\text { unadjusted for clustering } \\
\text { effects }\end{array}$ & $\begin{array}{l}\text { Intraclass } \\
\text { correlation }\end{array}$ & $\begin{array}{l}\text { Cluster-adjusted } \\
\text { IRR ( } 95 \% \text { confidence } \\
\text { limits) }\end{array}$ & $\begin{array}{l}\text { Post hoc power for } \\
\text { the cluster-adjusted } \\
\text { IRR }\end{array}$ \\
\hline \multicolumn{8}{|c|}{ All injuries } \\
\hline NMC & 335 & 12790 & $26.2(23.5,29.2)$ & \multirow{3}{*}{$\begin{array}{l}0.93(0.81,1.07) \\
p=0.31\end{array}$} & \multirow[t]{3}{*}{0.0117} & \multirow{3}{*}{$\begin{array}{l}0.92(0.68,1.23) \\
p=0.57\end{array}$} & \multirow[t]{3}{*}{$9 \%$} \\
\hline Control & 438 & 15537 & $28.2(25.6,31.0)$ & & & & \\
\hline All & 773 & 28327 & $27.3(25.4,29.3)$ & & & & \\
\hline \multicolumn{8}{|c|}{ Lower limb (knee, ankle, lower leg and thigh) } \\
\hline NMC & 151 & 12790 & $11.8(10.0,13.8)$ & \multirow{4}{*}{$\begin{array}{l}0.80(0.65,0.99) \\
p=0.038\end{array}$} & \multirow[t]{3}{*}{0.0007} & \multirow{3}{*}{$\begin{array}{l}0.78(0.56,1.08) \\
p=0.14\end{array}$} & \multirow[t]{3}{*}{$45 \%$} \\
\hline Control & 228 & 15537 & $14.7(12.8,16.7)$ & & & & \\
\hline All & 379 & 28327 & $13.4(12.1,14.8)$ & & & & \\
\hline \multicolumn{7}{|l|}{ Knee } & \\
\hline NMC & 32 & 12790 & $2.5(1.7,3.5)$ & \multirow{3}{*}{$\begin{array}{l}0.65(0.42,0.99) \\
p=0.046\end{array}$} & \multirow[t]{3}{*}{0.0132} & \multirow{3}{*}{$\begin{array}{l}0.50(0.24,1.05) \\
p=0.07\end{array}$} & \multirow[t]{3}{*}{$60 \%$} \\
\hline Control & 60 & 15537 & $3.9(2.9,5.0)$ & & & & \\
\hline All & 92 & 28327 & $3.2(2.6,4.0)$ & & & & \\
\hline
\end{tabular}


injuries, commonly seen in the sport. ${ }^{22}$ Our NMC programme was designed based on detailed knowledge of the LLIs sustained in men in $\mathrm{AF}^{8}{ }^{9} 15$ mechanisms for some of these LLIs (eg, anterior cruciate ligament injuries $(\mathrm{ACL})^{30-35}$ ), and training measures that successfully addressed the mechanisms in laboratory based RCTs. ${ }^{3-38}$ The intervention design also borrowed from previous studies that have been shown to be effective in preventing knee injuries specifically and LLIs in general. ${ }^{39-41}$ As a result, the specific components of the programme were well targeted for the target population and could be readily adopted in community AF training sessions. ${ }^{17}{ }^{24}$ The developed NMC programme also has the potential to be transferable to other sports requiring change of direction, balance and landing tasks as skills to reduce injury risk. ${ }^{21} 39-41$

In addition to high reliability and completeness of data collection, ${ }^{19}$ this cRCT has a number of other strengths. The group randomisation covered multiple clubs in different geographical locations that are representative of players in community AF. Injury rates are known to vary at different times of the season so data were collected over the entire preseason and playing season. Community AF players often join or leave a club at any stage so our criteria allowed inclusion up to 13 weeks, covering the preseason and start of the competition season, to enable time for players to be included in the trial, whether it was the NMC or the control arm. Injury rates were calculated on a per exposure basis, thereby accounting for players who were not present at every game or training session and, as such, did not contribute to the group of players at risk of injury. Allocation to the NMC and the control trial arms was blinded to PDCs, players, data coders and data analysts. This means that the PAFIX cRCT was as close as possible to a true double-blind trial, which is seldom reported in sports injury prevention studies. Finally, this study has adopted a very high standard of methodological reporting and analysis, which demonstrates the need to cluster-adjust results and report post hoc power calculations.

A limitation of this study is that the preseason baseline survey, aimed at collecting player characteristic information (eg, playing history, level of play, age and previous injury history), was only administered to, and completed by, around $50 \%$ of players. This was the only time where date of birth was recorded, so players' were excluded from the analysis when their age could not be confirmed. Likewise, comparison of pre-existing injuries was available only for the same, non-random subset of players. Although there is a lack of baseline data concerning the players' general health and injury history, the wide sampling of players from 18 teams in two different states means that the included

\section{What is already known on this subject}

- Australian football players have a high risk of injury, particularly to the lower limb, and preventing these injuries is important for players' participation and long-term health.

- The primary types of lower limb injuries sustained by Australian football players have been established and the mechanisms of these lower limb injuries, particularly for the knee, have been ascertained.

- Exercise-based injury prevention programmes are a potentially useful way of delivering injury prevention benefits to sports participants although investigations of their real-world effectiveness are lacking.

\section{What this study adds}

- This is the first study to report on the effectiveness of any injury prevention programme in community level Australian football for male players.

- Robust information on the rate of injuries in Australian football games and a demonstration of the importance of appropriate cluster adjustment has been presented.

- The rate of knee injuries and other lower limb injuries in Australian football games can be lowered by employing a specific programme of neuromuscular training exercises into regular training sessions.

players are likely to be an accurate representation of AF players in community leagues across Australia.

In conclusion, this ITT analysis indicates that clinically relevant reduced knee injury and LLI rates can be achieved through targeted exercise training programmes in men's community AF. The results should be considered alongside a range of additional issues including player compliance with the programme, since the preventive effect of the current programme was probably affected by low rates of programme reach and adoption. ${ }^{27}$ However, this study adds value to the growing evidence in support of targeted exercise training programmes for injury reductions in community level sport.

Twitter Follow Caroline Finch at @CarolineFinch, Lauren Fortington at @LFortington and Muhammad Akram at @Akers0071, Tim Doyle at @tladoyle, Dara Twomey at @daratwomey1

Acknowledgements The authors thank the staff in the Victorian Country Football League and Western Australian Amateur Football League who facilitated the PAFIX study within their competitions. The authors also thank club officials and team coaches/trainers for assistance, and the players who gave of their time to be trained by the PAFIX study personnel.

Contributors $A$ contribution to conception and design (CFF, BCE and DGL), analysis and interpretation of data (CFF, DGL, DT, BCE, TLAD, LVF and MA), and drafting (LVF and CFF) and reviewing (DT, BCE, DGL, TLAD and MA) the paper critically for important intellectual content has been met by all authors. All authors have given final approval of the version submitted for publication.

Funding The PAFIX study was funded by a National Health and Medical Research Council (NHMRC) grant (ID400937) to CFF, DGL and BCE; Western Australian Medical Health and Research Infrastructure Fund to DGL; and the University of Western Australia Research Grants Scheme to TLAD. CFF is funded by a NHMRC Principal Research Fellowship (ID1058737). ACRISP is one of the International Research Centres for the Prevention of Injury and Protection of Athlete Health supported by the International Olympic Committee (IOC).

Competing interests None declared.

Ethics approval The University of Ballarat Human Research Ethics Committee and the University of Western Australia Research Ethics Committee.

Provenance and peer review Not commissioned; externally peer reviewed.

Open Access This is an Open Access article distributed in accordance with the Creative Commons Attribution Non Commercial (CC BY-NC 4.0) license, which permits others to distribute, remix, adapt, build upon this work non-commercially, and license their derivative works on different terms, provided the original work is properly cited and the use is non-commercial. See: http://creativecommons.org/ licenses/by-nc/4.0/

\section{REFERENCES}

1 Verhagen E, Finch CF. Setting our minds to implementation. Br J Sports Med 2011;45:1015-6.

2 Finch $\mathrm{CF}$, Donaldson $\mathrm{A}$. A sports setting matrix for understanding the implementation context for community sport. Br J Sports Med 2010;44:973-8.

3 Andrew N, Gabbe BJ, Cook J, et al. Could targeted exercise programmes prevent lower limb injury in community Australian football? Sports Med 2013;43:751-63. 
4 Stojanovic MD, Ostojic SM. Preventing ACL injuries in team-sport athletes: a systematic review of training interventions. Res Sports Med 2012;20:223-38.

5 Dai $B$, Herman D, Liu $H$, et al. Prevention of ACL injury, part II: effects of ACL injury prevention programs on neuromuscular risk factors and injury rate. Res Sports Med 2012;20:198-222.

6 Alentorn-Geli E, Mendiguchia J, Samuelsson K, et al. Prevention of non-contact anterior cruciate ligament injuries in sports. Part II: systematic review of the effectiveness of prevention programmes in male athletes. Knee Surg Sports Traumatol Arthrosc 2014;22:16-25.

7 Cassell EP, Finch CF, Stathakis VZ. Epidemiology of medically treated sport and active recreation injuries in the Latrobe Valley, Victoria, Australia. Br J Sports Med 2003:37:405-9.

8 Finch CF, Da Costa A, Stevenson M, et al. Sports injury experiences from the Western Australian sports injury cohort study. Aust NZ J Public Health 2002;26: 462-7.

9 Finch $C F$, Gabbe BJ, White PE, et al. Priorities for investment in injury prevention in community Australian football. Clin J Sport Med 2013;23:430-8.

10 Ekegren CL, Gabbe BJ, Finch CF. Medical-attention injuries in community Australian football: a review of 30 years of surveillance data from treatment sources. Clin J Sport Med 2015;25:162-72.

11 Ekegren CL, Gabbe BJ, Finch CF. Injury surveillance in community sport: can we obtain valid data from sports trainers? Scand J Med Sci Sports 2015;25:315-22.

12 Lohmander LS, Englund PM, Dahl LL, et al. The long-term consequence of anterior cruciate ligament and meniscus injuries: osteoarthritis. Am J Sports Med 2007;35:1756-69.

13 Caine DJ, Golightly YM. Osteoarthritis as an outcome of paediatric sport: an epidemiological perspective. Br J Sports Med 2011;45:298-303.

14 Finch CF, Kemp JL, Clapperton AJ. The incidence and burden of hospital-treated sports-related injury in people aged 15+ years in Victoria, Australia, 2004-2010: a future epidemic of osteoarthritis? Osteoarthr Cartilage 2015;23:1138-43.

15 Finch CF, Lloyd DG, Elliott BC. The Preventing Australian Football Injuries with Exercise (PAFIX) Study: a group randomised controlled trial. Inj Prev 2009;15:e1.

16 Altman DG, Schulz KF, Moher D, et al. The revised CONSORT statement for reporting randomized trials:explanation and elaboration. Ann Intern Med 2001;134:663-94.

17 Finch CF, Doyle TLA, Dempsey AR, et al. What do community football players think about different exercise-training programmes? Implications for the delivery of lower limb injury prevention programmes. Br J Sports Med 2014;48:702-7.

18 Hollis SJ, Campbell F. What is meant by intention to treat analysis? Survey of published randomised controlled trials. BMJ 1999;319:670-4.

19 Twomey DM, Finch CF, Doyle TL, et al. Level of agreement between field-based data collectors in a large scale injury prevention randomised controlled trial. I Sci Med Sport 2011;14:121-5.

20 Hayes RJ, Bennett $\mathrm{S}$. Simple sample size calculation for cluster-randomized trials. Int J Epidemiol 1999;28:319-26.

21 Sugimoto D, Myer G, Micheli L, et al. ABCs of evidence-based anterior cruciate ligament injury prevention strategies in female athletes. Curr Phys Med Rehabil Rep 2015:3:43-9.

22 ter Stege MP, Dallinga J, Benjaminse A, et al. Effect of interventions on potential, modifiable risk factors for knee injury in team ball sports: a systematic review. Sports Med 2014;44:1403-26.
23 Herman K, Barton C, Malliaras P, et al. The effectiveness of neuromuscular warm-up strategies, that require no additional equipment, for preventing lower limb injuries during sports participation: a systematic review. BMC Medicine 2012;10:75.

24 Grimm NL, Jacobs JC, Kim J, et al. Anterior cruciate ligament and knee injury prevention programs for soccer players: a systematic review and meta-analysis. Am J Sports Med 2015:2049-56.

25 Soligard T, Nilstad A, Steffen K, et al. Compliance with a comprehensive warm-up programme to prevent injuries in youth football. Br J Sports Med 2010;44:787-93.

26 Steffen K, Emery CA, Romiti M, et al. High adherence to a neuromuscular injury prevention programme (FIFA 11+) improves functional balance and reduces injury risk in Canadian youth female football players: a cluster randomised trial. $\mathrm{Br} J$ Sports Med 2013;47:794-802.

27 Finch CF, Diamantopoulou K, Twomey DM, et al. The reach and adoption of a coach-led exercise training programme in community football. Br J Sports Med 2014;48:718-23.

28 Verhagen EALM, Hupperets MDW, Finch CF, et al. The impact of adherence on sports injury prevention effect estimates in randomised controlled trials: Looking beyond the CONSORT statement. J Sci Med Sport 2011;14:287-92.

29 Fortington LV, Donaldson A, Lathlean T, et al. When 'just doing it' is not enough: assessing the fidelity of player performance of an injury prevention exercise program. J Sci Med Sport 2015;18:272-7.

30 Besier TF, Lloyd DG, Ackland TR, et al. Anticipatory effects on knee joint loading during running and cutting maneuvers. Med Sci Sports Exerc 2001;33:1176-81.

31 Besier TF, Lloyd DG, Cochrane JL, et al. External loading of the knee joint during running and cutting maneuvers. Med Sci Sports Exerc 2001;33:1168-75.

32 Cochrane JL, Lloyd DG, Buttfield A, et al. Characteristics of anterior cruciate ligament injuries in Australian football. J Sci Med Sport 2007;10:96-104.

33 Dempsey AR, Lloyd DG, Elliott BC, et al. Whole body kinematics and knee moments that occur during an overhead catch and landing task in sport. Clin Biomech 2012;27:466-74.

34 Dempsey AR, Lloyd DG, Elliott BC, et al. The effect of technique change on knee loads during sidestep cutting. Med Sci Sports Exerc 2007;39:1765-73.

35 Donnelly CJ, Lloyd DG, Elliott BC, et al. Optimizing whole body kinematics to minimize valgus knee loading during sidestepping: implications for $A C L$ injury risk. J Biomechs 2012;45:1491-7.

36 Cochrane JL, Lloyd DG, Besier TF, et al. Training affects knee kinematics and kinetics in cutting maneuvers in sport. Med Sci Sports Exerc 2010;42:1535-44.

37 Dempsey AR, Lloyd DG, Elliott BC, et al. Can technique modification training reduce knee loading in landing tasks? J App/ Biomech 2014;30:231-6.

38 Dempsey AR, Lloyd DG, Elliott BC, et al. Changing sidestep cutting technique reduces knee valgus loading. Am J Sports Med 2009;37:2194-200.

39 Hewett TE, Lindenfeld TN, Riccobene JV, et al. The effect of neuromuscular training on the incidence of knee injury in female athletes. A prospective study. Am J Sports Med 1999;27:699-706.

40 Mandelbaum BR, Silvers HJ, Watanabe DS, et al. Effectiveness of a neuromuscular and proprioceptive training program in preventing anterior cruciate ligament injuries in female athletes: 2-year follow-up. Am J Sports Med 2005;33: 1003-10.

41 Myklebust G, Engebretsen L, Braekken IH, et al. Prevention of anterior cruciate ligament injuries in female team handball players: a prospective intervention study over three seasons. Clin J Sport Med 2003;13:71-8.

ImPACT is a model for concussion management that works with New York schools' insurance companies to support a comprehensive head injury prevention programme. The programme helps manage sports injuries and trains staff to recognise, respond to and prevent concussions. Comment: the role of insurance companies in prevention should not be overlooked. 